\title{
Quasi-Exact Solvability and the direct approach to invariant subspaces
}

\author{
D. Gómez-Ullate* N. Kamran, ${ }^{\dagger}$ and R. Milson ${ }^{\ddagger}$
}

January 20, 2004

\begin{abstract}
We propose a more direct approach to constructing differential operators that preserve polynomial subspaces than the one based on considering elements of the enveloping algebra of $\mathfrak{s l}(2)$. This approach is used here to construct new exactly solvable and quasi-exactly solvable quantum Hamiltonians on the line which are not Lie-algebraic. It is also applied to generate potentials with multiple algebraic sectors. We discuss two illustrative examples of these two applications: an interesting generalization of the Lamé potential which posses four algebraic sectors, and a quasi-exactly solvable deformation of the Morse potential which is not Lie-algebraic.
\end{abstract}

\section{Introduction}

Our purpose in this paper is to show that the property of quasi-exact solvability for Schrödinger operators admits an effective formulation which goes beyond the Lie algebraic context of hidden symmetry algebras, and to show explicitly that several classes of physically relevant potentials are quasi-exactly solvable in this more general sense. These include novel generalizations of the Lamé and Morse potentials.

\footnotetext{
*CRM, Montreal, Canada. E-mail: ullate@crm.umontreal.ca

${ }^{\dagger}$ McGill University, Montreal, Canada. . E-mail: nkamran@math.mcgill.ca

${ }_{\ddagger}^{\ddagger}$ Dalhouise University, Halifax, Canada. E-mail: milson@mathstat.dal.ca
} 
To put our results in context, we begin by briefly recalling the general definition of quasi-exact solvability, as well as the Lie algebraic formulation which applies to some particular classes of invariant subspaces.

Consider a one-dimensional Schrödinger operator

$$
H=-\partial_{x x}+U(x),
$$

which is assumed to be essentially self-adjoint on a Hilbert space $\mathcal{H}$ of wave functions. We say that $H$ is quasi-exactly solvable (QES) $[16,19]$ if it leaves invariant a non-trivial finite-dimensional subspace $\mathcal{M} \subset \mathcal{H}$

$$
H \mathcal{M} \subset \mathcal{M}
$$

where $\mathcal{M}$ is

$$
\mathcal{M}=\operatorname{span}\left\{\phi_{1}, \ldots, \phi_{n}\right\}, \quad \phi_{i} \in \mathcal{H} .
$$

In this case the restriction of $H$ to $\mathcal{M}$ is represented by a finite dimensional matrix, and a finite portion of the spectrum of $H$ can be obtained algebraically by diagonalizing this matrix. If $H$ actually preserves a complete flag of subspaces

$$
\mathcal{M}_{1} \subset \mathcal{M}_{2} \subset \cdots \subset \mathcal{M}_{n} \subset \ldots
$$

then it is said to be exactly solvable (ES). Given a certain potential $U(x)$ it is in general very difficult to determine whether $H$ preserves some non-trivial invariant subspace $\mathcal{M}$. One generally proceeds in the opposite direction, and starting from a known finite-dimensional subspace $\mathcal{M}$ one attempts to determine all Hamiltonians $H$ which are known a priori to leave $\mathcal{M}$ invariant. In most applications $\mathcal{M}$ is assumed to be of the form

$$
\begin{aligned}
\mathcal{M}_{n} & =\mu(x) \mathcal{P}_{n}(z(x)), \\
\mathcal{P}_{n} & =\left\langle 1, z, z^{2}, \ldots, z^{n}\right\rangle,
\end{aligned}
$$

where $\mu$ is some smooth non-vanishing multiplier, so that the first problem is to construct second-order differential operators which leave the polynomial module $\mathcal{P}_{n}$ invariant. In this context, Burnside's theorem ensures that every operator which leaves $\mathcal{P}_{n}$ invariant is an element of the enveloping algebra of the Lie algebra $\mathfrak{s l}(2)$ in the following standard representation

$$
J_{n}^{+}=z^{2} \partial_{z}-n z, \quad J_{n}^{0}=z \partial_{z}-\frac{n}{2}, \quad J_{n}^{-}=\partial_{z} .
$$


This approach is known as the Lie algebraic approach and $\mathfrak{s l}(2)$ is said to be a hidden symmetry algebra of the Hamiltonian. The Lie-algebraic approach has been extended to higher dimensional problems: some examples of Liealgebraic Hamiltonians in two dimensions are given in [13], while the quantum $N$-body Calogero-Moser model has been shown to have hidden symmetry algebra $\mathfrak{s l}(N+1)$ [20], and many-body problems with elliptic interaction were shown to be Lie-algebraic in [8].

In the last twenty five years, most of the papers dealing with quasi-exact solvability have made use of the Lie-algebraic approach, using irreducible representations of finite-dimensional Lie algebras by first-order differential operators as building blocks for constructing differential operators with invariant subspaces. Despite the success of this method, there remain some natural questions which are not amenable to this Lie-algebraic approach. For example, one would like to be able to construct the most general differential operator that will map a polynomial module $\mathcal{P}_{n}$ into a proper subspace $\mathcal{P}_{n-k} \subset \mathcal{P}_{n}$ or to know what are the operators that preserve monomial subspaces, that is subspaces of $\mathcal{P}_{n}$ which admit a monomial basis, but where all degrees up to $n$ need not be present (see definition (5) in Section 2). It is the latter question that we consider in Section 2 of this paper, where we obtain a general structure theorem for the space of linear differential operators preserving a general monomial subspace. Much of the contents of this Section can already be found in a paper by Post and Turbiner in [18]. Sections 3 and 4 form the heart of our paper. Section 3 consists in an explicit application of the results of Section 2 to Hamiltonians with multiple algebraic sectors viewed from the perspective of monomial subspaces/ This Section also includes an intriguing generalization of the Lamé equation which is shown to admit four algebraic sectors instead of the double algebraic sector admitted by the classical Lamé equation. In Section 4 we discuss a modification of the Morse potential which is shown to preserve a proper monomial subspace of $\mathcal{P}_{n}$. This class of potentials cannot be obtained in the Lie-algebraic approach and therefore they are not present in the classification of Lie-algebraic potentials on the line [11]. 


\section{The direct approach to invariant monomial subspaces}

In this Section we shall introduce the concept of a monomial subspace and we will characterize all differential operators of order $r$ that preserve a given monomial subspace. This Section follows essentially the algebraic approach introduced in [18].

For a finite subset $I \subset \mathbb{Z}$ let us define a monomial subspace $\mathcal{P}_{I}$ as

$$
\mathcal{P}_{I}=\operatorname{span}\left\{z^{k}: k \in I\right\},
$$

and let $N=|I|$ be the dimension of $\mathcal{P}_{I}$. A non-trivial differential operator $T_{r}^{d}$ of order $r$ will be said to be of degree $d \in \mathbb{Z}$ if and only if

$$
T_{r}^{d}\left[x^{j}\right]=c_{j} x^{j+d} \quad \forall j \in \mathbb{Z} .
$$

Let us denote by $\mathfrak{D}_{r}\left(\mathcal{P}_{I}\right)$ the space of differential operators of degree $r$ with analytic coefficients that preserve $\mathcal{P}_{I}$.

Proposition 1 Every linear transformation of $\mathcal{P}_{I}$ can be represented by an element of $\mathfrak{D}_{N-1}\left(\mathcal{P}_{I}\right)$.

Proof. A basis of the dual space is given by the following $(N-1)$-th order operators

$$
L_{k}=z^{-k} \prod_{\substack{j \in I \\ j \neq k}} \frac{z \partial_{z}-j}{k-j}, \quad k \in I,
$$

which clearly satisfy $L_{k}\left[z^{j}\right]=\delta_{k}^{j}, \forall j, k \in I$. Therefore, every element of $\operatorname{End}\left(\mathcal{P}_{I}\right)$ can be represented by an operator of the form

$$
T_{N-1}=\sum_{j, k \in I} a_{j k} z^{j} L_{k} \in \mathfrak{D}_{N-1}\left(\mathcal{P}_{I}\right)
$$

QED

In fact, these operator duals can be constructed for more general subspaces of functions (not just polynomials) [15], but the order of the differential operators grows with the dimension of the space. We are interested in constructing differential operators that preserve $\mathcal{P}_{I}$ of order $r<N-1$. Let us denote by $\mathfrak{D}_{r}^{d}\left(\mathcal{P}_{I}\right) \subseteq \mathfrak{D}_{r}\left(\mathcal{P}_{I}\right)$ the space of operators of order $r$ and degree $d$ that preserve $\mathcal{P}_{I}$. In order to characterize $\mathfrak{D}_{r}\left(\mathcal{P}_{I}\right)$ we can concentrate in operators having a fixed degree, since the following Proposition holds. 


\section{Proposition 2}

$$
\mathfrak{D}_{r}\left(\mathcal{P}_{I}\right)=\bigoplus_{d} \mathfrak{D}_{r}^{d}\left(\mathcal{P}_{I}\right)
$$

proof Any operator $T \in \mathfrak{D}_{r}\left(\mathcal{P}_{I}\right)$ can be decomposed uniquely as a sum $T=\sum_{d} T^{(d)}$ of operators of fixed degree. Then it suffices to prove that if $T$ preserves $\mathcal{P}_{I}$ then each $T^{(d)}$ also preserves $\mathcal{P}_{I}$. Since $T \mathcal{P}_{I} \subset \mathcal{P}_{I}$, then

$$
T\left[z^{k}\right]=\sum_{j \in I} a_{j k} z^{j}, \quad k \in I,
$$

and it follows that

$$
T^{(d)}\left[z^{k}\right]= \begin{cases}a_{d+k, k} z^{d+k}, & \text { if } d+k \in I ; \\ 0 & \text { otherwise. }\end{cases}
$$

which proves that $T^{(d)} \mathcal{P}_{I} \subset \mathcal{P}_{I}$. QED

Let us define the primitive index sets

$$
I(a, b, c)=\{a+k c: 0 \leq k \leq b\}
$$

and the primitive monomial subspaces $\mathcal{P}_{a, b, c}$ as

$$
\mathcal{P}_{a, b, c}=\mathcal{P}_{I(a, b, c)}=\operatorname{span}\left\{z^{a+k c}: 0 \leq k \leq b\right\}
$$

where $a$ is the smallest exponent, $c$ is the size of the gap and $b+1$ is the number of elements. In fact,

$$
\mathcal{P}_{a, b, c}=z^{a} \mathcal{P}_{b}\left(z^{c}\right),
$$

where $\mathcal{P}_{n}(z)=\left\langle 1, z, \ldots, z^{n}\right\rangle$ denotes the full polynomial module (no gaps). Every monomial subspace can be trivially decomposed as a direct sum of singleton primitive monomial subspaces

$$
\mathcal{P}_{I}=\bigoplus_{a \in I} \mathcal{P}_{a, 0,0},
$$

which corresponds to representing $I$ as the union of $N$ singletons. The differential operators (77) of order $N-1$ are constructed to annihilate $N-1$ different exponents. However, it is precisely when the index set $I$ can be expressed as the union of a smaller number of primitive index sets (9), that lower order operators preserving $\mathcal{P}_{I}$ can exist. This consideration leads to the following key theorem: 
Theorem 1 If the set I can be expressed as the union of a finite number of primitive index sets of the form

$$
I=\bigcup_{i=1}^{r} I\left(a_{i}, b_{i}, d\right),
$$

then there exist operators $T_{r}^{( \pm d)}$ of order $r$ and degree $\pm d$ that preserve $\mathcal{P}_{I}$. Conversely, if $T_{r}^{(d)}$ is an operator of order $r<N$ and degree $d>0$ that preserves a given monomial subspace $\mathcal{P}_{I}$, then there exists a $T_{r}^{(-d)}$ which also preserves $\mathcal{P}_{I}$ and a vector-space decomposition of $\mathcal{P}_{I}$ into primitive monomial subspaces

$$
\mathcal{P}_{I}=\sum_{i=1}^{r} \mathcal{P}_{a_{i}, b_{i}, d}
$$

Proof. Suppose that (11) holds and consider the following $r$-th order operators

$$
\begin{aligned}
T_{r}^{(-d)} & =z^{-d} \prod_{i=1}^{r}\left(z \partial_{z}-a_{i}\right) \\
T_{r}^{(d)} & =z^{d} \prod_{i=1}^{r}\left(z \partial_{z}-\left(a_{i}+d b_{i}\right)\right)
\end{aligned}
$$

The lowering operator maps $z^{a_{i}+k d}$ to $z^{a_{i}+(k-1) d}$ and annihilates $z^{a_{i}}$ for all $i=$ $1, \ldots, r$. Similarly the raising operator maps $z^{a_{i}+k d}$ to $z^{a_{i}+(k+1) d}$ annihilates $z^{a_{i}+d b_{i}}$ for all $i$. It is clear then that

$$
T_{r}^{( \pm d)} \mathcal{P}_{I} \subset \mathcal{P}_{I} .
$$

To prove the converse we assume that $T^{(d)}$ is an operator of degree $d>0$ and order $r$ that preserves a given $\mathcal{P}_{I}$. The operator $z^{-d} T^{(d)}$ has degree 0 , and hence has a unique factorization of the form

$$
z^{-d} T^{(d)}=c \prod_{i=1}^{r}\left(z \partial_{z}-k_{i}\right),
$$

up to a multiplicative constant $c$. For each $i$, let $b_{i}$ to be the largest possible natural number such that $I\left(a_{i}, b_{i}, d\right) \subset I$, where $a_{i}=k_{i}-d b_{i}$. 
If (111) did not hold, suppose that $\alpha$ is the largest element of $I$ that is not in any of the $I\left(a_{i}, b_{i}, d\right)$. Since $\alpha \neq k_{i}$ for all $i, T^{(d)}$ does not annihilate $z^{\alpha}$ and by assumption $\alpha+d \in I$. But since $\alpha$ was the largest element in $I$ which does not belong to any of the $I\left(a_{i}, b_{i}, d\right)$, we must have $\alpha+d \in I\left(a_{i}, b_{i}, d\right)$ for some $i$, but then by the way $b_{i}$ was chosen, $\alpha \in I$ as well, which is a contradiction. QED

\subsection{Second order operators}

The case of second order operators is particularly important for the applications in quantum mechanics, and we shall concentrate to their study in this Section. From Theorem 11 we know that if a given monomial subspace $\mathcal{P}_{I}$ is invariant under $T_{2}^{(d)}$, it is also invariant under $T_{2}^{(-d)}$, and of course it always happens that $T_{2}^{(0)} \in \mathfrak{D}_{2}\left(\mathcal{P}_{I}\right)$ for any monomial subspace $\mathcal{P}_{I}$. The natural question is now to explore whether a given monomial subspace can be preserved by several operators of different degrees.

Theorem 2 Suppose that a monomial subspace $\mathcal{P}_{I}$ of dimension $|I|>3$ is invariant under two second order operators $T_{2}^{\left(d_{1}\right)}$ and $T_{2}^{\left(d_{2}\right)}$ of degrees $d_{1}, d_{2} \in$ $\mathbb{Z}^{+}$with $d_{1}>d_{2}$ coprimes. Then $d_{1}=2, d_{2}=1$ and $\mathcal{P}_{I}$ must be one of the following 3 modules:

i) $\mathcal{P}_{n}=\left\langle 1, z, z^{2}, \ldots, z^{n}\right\rangle$

ii) $\tilde{\mathcal{P}}_{n}=\left\langle 1, z, z^{2}, \ldots, z^{n-2}, z^{n}\right\rangle$

iii) $\hat{\mathcal{P}}_{n}=\left\langle 1, z^{2}, \ldots, z^{n}\right\rangle$

Proof. Let $I=\left\{i_{1}, \ldots, i_{N}\right\}$ with $i_{1}<i_{2}<\cdots<i_{N-1}<i_{N}$. Both $T_{2}^{\left(d_{1}\right)}$ and $T_{2}^{\left(d_{2}\right)}$ must annihilate $z^{i_{N}}$, so they must be of the form

$$
\begin{aligned}
& T_{2}^{\left(d_{1}\right)}=z^{d_{1}}\left(z \partial_{z}-i_{N}\right)\left(z \partial_{z}-a_{1}\right) \\
& T_{2}^{\left(d_{2}\right)}=z^{d_{2}}\left(z \partial_{z}-i_{N}\right)\left(z \partial_{z}-a_{2}\right)
\end{aligned}
$$

with $a_{1}$ and $a_{2}$ still to be specified. The difference $i_{N}-i_{N-1}$ must be equal to either $d_{1}$ or $d_{2}$ because otherwise both $T_{2}^{\left(d_{1}\right)}$ and $T_{2}^{\left(d_{2}\right)}$ must annihilate $z^{i_{N-1}}$ too, fixing $a_{1}=a_{2}=i_{N-1}$. But then either $T_{2}^{\left(d_{1}\right)}\left[z^{i_{N-2}}\right]$ or $T_{2}^{\left(d_{2}\right)}\left[z^{i_{N-2}}\right]$ will be outside $\mathcal{P}_{I}$. So two possibilities remain 
1. $i_{N}-i_{N-1}=d_{1}$

In this case $T_{2}^{\left(d_{2}\right)}$ must annihilate $z^{i_{N-1}}$ so $a_{2}=i_{N-1}$, but this forces $i_{N-1}-i_{N-2}=d_{2}$ since otherwise $T_{2}^{\left(d_{2}\right)}\left[z^{i_{N-2}}\right] \notin \mathcal{P}_{I}$. It follows that $T_{2}^{\left(d_{1}\right)}\left[z^{i_{N-2}}\right]=0$ fixing $a_{1}=i_{N-2}$. But now neither $T_{2}^{\left(d_{2}\right)}$ nor $T_{2}^{\left(d_{1}\right)}$ can annihilate $z^{i_{N-3}}$, so $i_{N-2}-i_{N-3}=d_{2}$ and $i_{N-1}-i_{N-3}=d_{1}$. This implies $d_{1}=2 d_{2}$ and since they are coprimes $d_{1}=2$ and $d_{2}=1$. The preserved module is then $\tilde{\mathcal{P}}_{n}$.

2. $i_{N}-i_{N-1}=d_{2}$

In this case $T_{2}^{\left(d_{1}\right)}\left[z^{i_{N-1}}\right]=0$ so $a_{1}=i_{N-1}$. The difference $i_{N-1}-i_{N-2}$ cannot be $d_{1}$ because otherwise $T_{2}^{\left(d_{2}\right)}$ would need to annihilate $z^{i_{N-2}}$, and then either $T_{2}^{\left(d_{1}\right)}\left[z^{i_{N-3}}\right]$ or $T_{2}^{\left(d_{2}\right)}\left[z^{i_{N-3}}\right] \notin \mathcal{P}_{I}$. So $i_{N-1}-i_{N-2}=d_{2}$ but then $T_{2}^{\left(d_{1}\right)}\left[z^{i_{N-2}}\right] \propto z^{i_{N}}$ and this can only happen if $d_{1}=2 d_{2}$. Iterating these arguments it is easy to see that all differences between successive indices must be equal to $d_{2}$ except for $i_{2}-i_{1}$ which could be either $d_{1}$ (which leads to $\hat{\mathcal{P}}_{n}$ ) or $d_{2}$, which leads the ordinary polynomial module $\mathcal{P}_{n}$. QED

Corollary 1 No monomial subspace $\mathcal{P}_{I}$ is invariant under three second order operators of relatively prime degrees $d_{1}, d_{2}, d_{3} \in \mathbb{Z}^{+}$.

Proof. It is clear that a third operator $T_{2}^{\left(d_{3}\right)}$ with an odd $d_{3}>2$ will not preserve any of the modules of the previous Theorem.

Corollary 2 The spaces of second order differential operators that leave $\mathcal{P}_{n}$, $\hat{\mathcal{P}}_{n}$ and $\tilde{\mathcal{P}}_{n}$ invariant have the following dimensions:

$$
\operatorname{dim} \mathfrak{D}_{2}\left(\mathcal{P}_{n}\right)=9, \quad \operatorname{dim} \mathfrak{D}_{2}\left(\hat{\mathcal{P}}_{n}\right)=\operatorname{dim} \mathfrak{D}_{2}\left(\tilde{\mathcal{P}}_{n}\right)=7
$$

Proof. For the case of $\mathcal{P}_{n}$, the dimensions of the corresponding operator spaces $\mathfrak{D}_{2}^{k}\left(\mathcal{P}_{n}\right)$ are:

$$
\operatorname{dim} \mathfrak{D}_{2}^{0}\left(\mathcal{P}_{n}\right)=3, \quad \operatorname{dim} \mathfrak{D}_{2}^{ \pm 1}\left(\mathcal{P}_{n}\right)=2, \quad \operatorname{dim} \mathfrak{D}_{2}^{ \pm 2}\left(\mathcal{P}_{n}\right)=1
$$


This follows from noting that the operators in question can be expressed in the form

$$
\begin{aligned}
T^{(0)} & =p\left(z \partial_{z}\right), \quad p(x) \in \mathcal{P}_{2}(x), \\
T^{(1)} & =z\left(a_{1} z \partial_{z}-b_{1}\right)\left(z \partial_{z}-n\right), \\
T^{(-1)} & =z^{-1}\left(a_{2} z \partial_{z}-b_{2}\right)\left(z \partial_{z}\right), \\
T^{(-2)} & =z^{-2}\left(z \partial_{z}-1\right)\left(z \partial_{z}\right), \\
T^{(2)} & =z^{2}\left(z \partial_{z}-n\right)\left(z \partial_{z}-(n-1)\right) .
\end{aligned}
$$

The situation for the case of $\hat{\mathcal{P}}_{n}$ is similar, the difference being that the dimension of $\mathfrak{D}_{2}^{ \pm 1}\left(\hat{\mathcal{P}}_{n}\right)$ is only one since the corresponding operators must have the form

$$
\begin{aligned}
T^{(1)} & =a_{1} z\left(z \partial_{z}-0\right)\left(z \partial_{z}-n\right), \\
T^{(-1)} & =a_{2} z^{-1}\left(z \partial_{z}-0\right)\left(z \partial_{z}-2\right) .
\end{aligned}
$$

Thus the dimension of $\mathfrak{D}_{2}\left(\hat{\mathcal{P}}_{n}\right)$ is seven. QEDIn order to apply this construction to Quantum Mechanics, we need to relate an operator $T \in \mathfrak{D}_{2}\left(\mathcal{P}_{I}\right)$ with a Schrödinger operator (11). This is always possible in one dimension by virtue of the following Lemma:

Lemma 1 Every second order operator $T$ of the form

$$
T=P(z) \partial_{z z}+\left(Q(z)+\frac{1}{2} P^{\prime}(z)\right) \partial_{z}+R(z),
$$

with $P(z)>0$ can be transformed into Schrödinger form (11) by a gauge transformation and a change of variables:

$$
H(x)=-\left.\mu(z) T(z) \mu^{-1}(z)\right|_{z=\xi^{-1}(x)},
$$

where

$$
\begin{aligned}
x & =\xi(z)=\int^{z} \frac{d \zeta}{\sqrt{P(\zeta)}} \\
\mu(z) & =\exp \int^{z} \frac{Q(\zeta)}{2 P(\zeta)} d \zeta
\end{aligned}
$$

The resulting potential $U(x)$ in the physical variable $x$ is given by

$$
U(x)=R(z)-\frac{1}{2} Q^{\prime}(z)+\left.\frac{Q(z)\left(P^{\prime}(z)-Q(z)\right)}{4 P(z)}\right|_{z=\xi^{-1}(x)}
$$




\section{Quantum Hamiltonians with multiple alge- braic sectors}

We are familiar with the fact that Schrödinger operators with even potentials will have eigenfunctions with well defined parity, and therefore the odd sector can be treated independently from the even sector. Let us look at the phenomenon of multiple algebraic sectors from the point of view of monomial subspaces. There are two mechanisms that can produce a multiple algebraic sector. The first one comes from the fact that a second order operator can preserve two different polynomial modules. From Theorem 1 we know that if $\mathfrak{D}_{2}^{(d)}\left(\mathcal{P}_{I}\right) \neq \emptyset$, then $\mathcal{P}_{I}$ can be decomposed as

$$
\mathcal{P}_{I}=\mathcal{P}_{a_{1}, n_{1}, d}+\mathcal{P}_{a_{2}, n_{2}, d}
$$

If in addition $\mathfrak{D}_{2}^{\left(d_{2}\right)}\left(\mathcal{P}_{I}\right)=\emptyset$ for every other $d_{2} \neq d, d_{2} \in \mathbb{Z}^{+}$then

$$
\begin{aligned}
& \mathfrak{D}_{2}^{(d)}\left(\mathcal{P}_{I}\right) \subset \mathfrak{D}_{2}^{(d)}\left(\mathcal{P}_{a_{1}, n_{1}, d}\right), \\
& \mathfrak{D}_{2}^{(d)}\left(\mathcal{P}_{I}\right) \subset \mathfrak{D}_{2}^{(d)}\left(\mathcal{P}_{a_{2}, n_{2}, d}\right),
\end{aligned}
$$

and both spaces are invariant separately. We can take $a_{1}=0$ without loss

of generality and let $p=a_{2} / d, p \in \mathbb{R}$. In the new variable $y=z^{d}$ the two invariant polynomial modules are

$$
\mathcal{M}_{1}=\mathcal{P}_{n_{1}}(y), \quad \mathcal{M}_{2}=y^{p} \mathcal{P}_{n_{2}}(y),
$$

and each of them is independently preserved.

Proposition 3 The most general second order differential operator that preserves both $\mathcal{M}_{1}$ and $\mathcal{M}_{2}$ in (23) is a linear combination of the following five operators

$$
\begin{aligned}
T_{2}^{(+1)} & =y\left(y \partial_{y}-n_{1}\right)\left(y \partial_{y}-\left(p+n_{2}\right)\right) \\
& =y^{3} \partial_{y y}+\left(1-p-n_{1}-n_{2}\right) y^{2} \partial_{y}+n_{1}\left(n_{2}+p\right) y \\
T_{2}^{(0)} & =y^{2} \partial_{y y} \\
T_{2}^{(-1)} & =y^{-1}\left(y \partial_{y}-0\right)\left(y \partial_{y}-p\right) \\
& =y \partial_{y y}+(1-p) \partial_{y} \\
T_{1}^{(0)} & =y \partial_{y} \\
T_{0}^{(0)} & =1
\end{aligned}
$$


These operators form a sub-family of the well known nine parameter family of Lie-algebraic second order operators that would preserve the polynomial module $\mathcal{M}_{1}$.

A second source of multiple algebraic sectors comes from noting that in Lemma 1 the correspondence between the algebraic operator $T$ and the Schrödinger operator $H$ is at least two to one:

Proposition 4 The operators $T_{1}$ and $T_{2}$ given by

$$
\begin{aligned}
& T_{1}=P(z) \partial_{z z}+\left(Q(z)+\frac{1}{2} P^{\prime}(z)\right) \partial_{z}+R(z) \\
& T_{2}=P(z) \partial_{z z}+\left(-Q(z)+\frac{3}{2} P^{\prime}(z)\right) \partial_{z}+R(z)-Q^{\prime}(z)+\frac{1}{2} P^{\prime \prime}(z)
\end{aligned}
$$

are both equivalent under the transformation (16) -(18) to the same Schrödinger operator $H=-\partial_{x x}+U(x)$ with potential $U(x)$ given by (19).

Therefore if both $T_{1}$ and $T_{2}$ preserve different finite dimensional subspaces, then the Schrödinger operator will have a double algebraic sector.

The two mechanisms described above can be combined to yield potentials with quadruple algebraic sectors. This will be the case if operators $T_{1}$ and $T_{2}$ in Proposition 4 are both a linear combination of the operators of Proposition 3. These ideas lead to a generalization of the Lamé potential that posses a quadruple algebraic sector.

\subsection{The generalized Lamé potential}

Let us consider the following linear combination of the operators (24)-(28):

$$
T=4 k T_{2}^{(+1)}+4(k+1) T_{2}^{(0)}+4 T_{2}^{(-1)}+4 b_{1} T_{1}^{(0)}
$$

which can be written in the form (15) with

$$
\begin{aligned}
& P(z)=4 z(z+1)(k z+1), \\
& Q(z)=-2 k\left(1+2 n_{1}+2 n_{2}+2 p\right) z^{2}+4\left(b_{1}-k-1\right) z+2-4 p, \\
& R(z)=4 k n_{1}\left(n_{2}+p\right) z .
\end{aligned}
$$

The cubic $P(z)$ determines the change of variables (17) and we must consider only the interval in $z$ such that $P(z)>0$ for the change of variables to be 
admissible. If we take $k<0$ and let $z \in(0,-1 / k)$ the change of variables determined by (17) is

$$
z=k^{-1} \operatorname{sn}^{2}\left(\sqrt{-k} x \mid k^{-1}\right)
$$

which maps the real line to the interval $(0,-1 / \mathrm{k})$. From equation (19) in Lema 1 we see that the condition for the potential to be nonsingular is that $Q(z)\left(P^{\prime}(z)-Q(z)\right)$ should divide $P(z)$, and since $z \in(0,-1 / k)$ this implies that

$$
Q(0)=0 \quad \text { or } \quad Q(0)=P^{\prime}(0)
$$

and

$$
Q(-1 / k)=0 \quad \text { or } \quad Q(-1 / k)=P^{\prime}(-1 / k) .
$$

The first of these conditions is satisfied when $p= \pm 1 / 2$ and since we can assume, without loss of generality, that 0 is the smallest power in the monomial subspace, then we must take $p=1 / 2$. The second set of conditions amounts to choosing $b_{1}$ as

$$
\begin{aligned}
& b_{1}=k-n_{1}-n_{2}, \\
& b_{1}=2 k-1-n_{1}-n_{2} .
\end{aligned}
$$

Therefore we can write the potential (19) after performing the change of variables (35) as

$$
V(z)=m l(l+1) \operatorname{sn}^{2}(x \mid m)-j(j+1) \operatorname{sn}^{2}\left(\sqrt{m-1} x \mid \frac{1}{1-m}\right)
$$

where $m=1-k$ and $j, l \in \mathbb{Z}$ are related to $n_{1}$ and $n_{2}$ as

$$
\begin{aligned}
l=n_{1}+n_{2}+1, & j=n_{1}-n_{2}-1 \\
l=n_{1}+n_{2}+2, & j=n_{2}-n_{1}
\end{aligned}
$$

depending on whether we use (36) or (37). Note that the particular cases $j=0$ and $j=-1$ correspond to the Lamé equation, and in those cases the two polynomial modules in (23) match with no half-integer gaps. However, for greater values of $j$ the potential departs from Lamé and develops an interesting shape, as can be seen in Figure 1. Depending on whether we use (36) or (37) in the formula for the gauge factor (18) we obtain

$$
\begin{aligned}
& \mu_{1}=(1+z)^{-l / 2}=\mathrm{cn}^{l}(x \mid m), \\
& \mu_{2}=(1+z)^{-l / 2}(1+k z)^{1 / 2}=\mathrm{cn}^{l}(x \mid m) \operatorname{cn}\left(\sqrt{-k} x \mid \frac{1}{1-m}\right),
\end{aligned}
$$




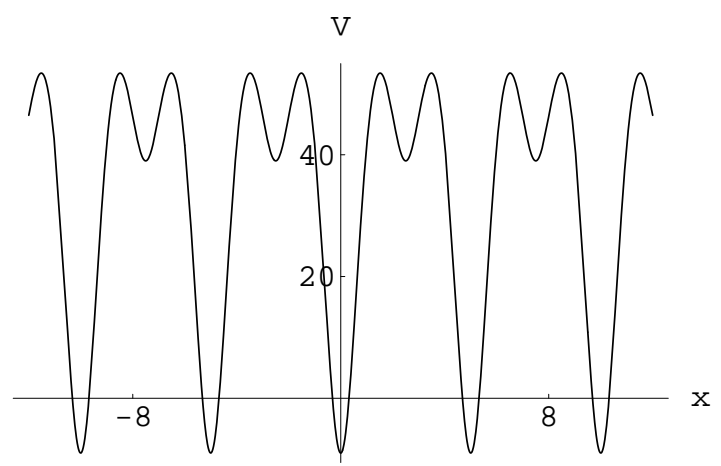

Figure 1: Generalized Lamé potential (38) for $m=11 / 10, l=9$ and $j=6$

To summarize the results in this section, we have proved that when $j$ and $l$ are integers of the same parity, the generalized Lamé potential (38) has the following quadruple algebraic sector:

$$
\begin{array}{ll}
\mathcal{M}_{1}^{(1)}=\mu_{1} \mathcal{P}_{n_{1}}(z), & \mathcal{M}_{2}^{(1)}=\mu_{1} \mathcal{P}_{n_{2}}(z), \\
\mathcal{M}_{1}^{(2)}=\mu_{2} \mathcal{P}_{\tilde{n}_{1}}(z), & \mathcal{M}_{2}^{(2)}=\mu_{2} \mathcal{P}_{\tilde{n}_{2}}(z),
\end{array}
$$

where

$$
\begin{array}{ll}
n_{1}=\frac{l+j}{2}, & n_{2}=\frac{l-j}{2}-1, \\
\tilde{n}_{1}=\frac{l-j}{2}-1, & \tilde{n}_{2}=\frac{l+j}{2}-1,
\end{array}
$$

and $z$ is given by (35). The total number of algebraic eigenfunctions is the sum of the dimensions of the four algebraic sectors, which equals $2 l+1$. It is quite remarkable that this number does not depend on $j$. In fact, when $j=0$ and $l \in \mathbb{N}$, these eigenfunctions are the well known $2 l+1$ Lamé polynomials. The period of the potential is $2 K$ where

$$
K=\int_{0}^{\frac{\pi}{2}} \frac{d \theta}{\sqrt{1-m^{2} \sin ^{2} \theta}}
$$

is the elliptic integral of the first kind. There are $l+1$ eigenfunctions which belong to the sectors $\mathcal{M}_{1,2}^{(1)}$ have the same period as the potential, while the $l$ eigenfuntions which belong to the sectors $\mathcal{M}_{1,2}^{(2)}$ have period $4 K$. A detailed study of the properties of these polynomial eigenfunctions shall be deferred to a forthcoming publication. 
Let us note as a conclusion that the generalized Lamé potential could be applied as realistic model of a one-dimensional crystal, which generalizes the models introduced by Alhassid et al. in [1]. The generalized Lamé potential would describe a one dimensional crystal with constant lattice spacing $a=2 K$ and two atoms of different atomic number. By changing the values of $j$ and $l$ the relative strength of the potential wells can be modified. The eigenvalues of the potential can be calculated algebraically and they describe the edges of the allowed energy bands of the crystal. Other possible applications of this potential include the quantum fluctuations of the inflaton field in certain cosmological models [6,14].

\section{New Quasi-exactly solvable hamiltonians which are not Lie-algebraic}

In this section we will apply a similar construction to obtain quasi-exactly solvable potentials that cannot be obtained as a quadratic polynomial in the generators of $\mathfrak{s l}(2)$. In order to construct second order operators that are not Lie-algebraic we should focus our attention in cases ii) and iii) of Theorem 2. the so called exceptional modules, since the full polynomial module $\mathcal{P}_{n}$ is the carrier space for an irreducible representation of $\mathfrak{s l}(2)$, and we have from Burnside's theorem that any operator which leaves $\mathcal{P}_{n}$ invariant must be a polynomial in the generators of $\mathfrak{s l}(2)$. Let us note that the two exceptional modules are projectively equivalent, since

$$
\hat{\mathcal{P}}_{n}(z)=z^{n} \tilde{\mathcal{P}}_{n}(1 / z),
$$

and we can therefore restrict our attention to one of them.

Proposition 5 A differential operator of order two or less preserves

$$
\hat{\mathcal{P}}_{n}(z)=\operatorname{span}\left\{1, z^{2}, \ldots, z^{n}\right\},
$$


if and only if it is a linear combination of the following seven operators:

$$
\begin{aligned}
T_{2}^{(+2)} & =z^{4} \partial_{z z}+2(1-n) z^{3} \partial_{z}+n(n-1) z^{2}, \\
T_{2}^{(+1)} & =z^{3} \partial_{z z}+(1-n) z^{2} \partial_{z} \\
T_{2}^{(0)} & =z^{2} \partial_{z z} \\
T_{2}^{(-1)} & =z \partial_{z z}-\partial_{z} \\
T_{2}^{(-2)} & =\partial_{z z}-2 z^{-1} \partial_{z} \\
T_{1}^{(0)} & =z \partial_{z} . \\
T_{0}^{(0)} & =1 .
\end{aligned}
$$

Proof. The sufficiency follows from the fact that each of the operators above preserves $\hat{P}_{n}$, which can be verified by direct computation. The necessity is a direct consequence of Corollary 2, and the fact that the above operators are linearly independent.

Consider the action of $S L(2, \mathbb{R})$ on the (projective) line according to the linear fractional, or Möbius transformations

$$
w \mapsto z=\frac{\alpha w+\beta}{\gamma w+\delta}, \quad \Delta=\alpha \delta-\beta \gamma=1 .
$$

There is an induced action of $S L(2)$ on the space $\mathcal{P}_{n}$ of polynomials of degree at most $n$, given by

$$
P(z) \mapsto \tilde{P}(w)=(\gamma w+\delta)^{n} P\left(\frac{\alpha w+\beta}{\gamma w+\delta}\right) .
$$

This irreducible multiplier representation of $S L(2)$ is isomorphic to the standard representation on homogeneous polynomials of degree $n$ in two variables. Since $\mathcal{P}_{n}$ is invariant under this transformation, it was used in [13] to classify the second order operators that preserve $\mathcal{P}_{n}$ into equivalence classes, each of them labeled by a simple canonical form. On the contrary, monomial subspaces (and in particular the exceptional module $\hat{\mathcal{P}}_{n}$ ) are not invariant under the transformation (50). This is clear since $\hat{\mathcal{P}}_{n} \subset \mathcal{P}_{n}$ is a hyperplane of co-dimension 1 in $\mathcal{P}_{n}$ which moves under the projective $S L(2)$ action. In particular, under the transformation (150) the exceptional module $\hat{\mathcal{P}}_{n}(z)$ transforms into

$$
\begin{aligned}
& \hat{\mathcal{P}}_{n}(z) \mapsto \mathcal{M}_{n}(w) \subset \mathcal{P}_{n}(w), \\
& \mathcal{M}_{n}(w)=\operatorname{span}\left\{(\gamma w+\delta)^{n-k}(\alpha w+\beta)^{k} \mid k=0,2, \ldots, n\right\} .
\end{aligned}
$$


It will be convenient to introduce a basis independent characterization of the space $\mathcal{M}_{n}$.

Proposition 6 If $\alpha, \gamma \neq 0$ a polynomial $P(w)$ of degree $n$ is in $\mathcal{M}_{n} \subset \mathcal{P}_{n}$ if and only if

$$
P^{\prime}(-\beta / \alpha)-n \alpha \gamma P(-\beta / \alpha)=0 .
$$

Proof. Since the condition is linear, it suffices to prove that it is satisfied by all the elements in the basis (51) of $\mathcal{M}_{n}(w)$. It is clear that all elements with $k \geq 2$ satisfy $P^{\prime}(-\beta / \alpha)=P(-\beta / \alpha)=0$ while the element with $k=0$ satisfies precisely condition (52). In fact, condition (152) is the transformation of $P^{\prime}(0)=0$ (which defines $\hat{\mathcal{P}}_{n}$ ) under the transformation (150). QED

We would now want to know which operators preserve the transformed monomial subspace $\mathcal{M}_{n}$. Of course if $T(z) \hat{\mathcal{P}}_{n}(z) \subset \hat{\mathcal{P}}_{n}(z)$ then

$$
\tilde{T}(w)=(\gamma w+\delta)^{n} \cdot T\left(\frac{\alpha w+\beta}{\gamma w+\delta}\right) \cdot(\gamma w+\delta)^{-n}
$$

will satisfy $\tilde{T}(w) \mathcal{M}_{n}(w) \subset \mathcal{M}_{n}(w)$. Transforming the operators of Proposition 5 under this prescription will lead to a basis of $\mathfrak{D}_{2}\left(\mathcal{M}_{n}\right)$. However, a simpler basis can be written in the form expressed by the following proposition:

Proposition 7 A basis of $\mathfrak{D}_{2}\left(\mathcal{M}_{n}\right)$ is given by the following seven operators:

$$
\begin{aligned}
& J_{1}(w)=(\alpha w+\beta)^{4} \partial_{w w}+2 \alpha(1-n)(\alpha w+\beta)^{3} \partial_{w}+n(n-1) \alpha^{2}(\alpha w+\beta)^{2}, \\
& J_{2}(w)=(\alpha w+\beta)^{3} \partial_{w w}+\alpha(1-n)(\alpha w+\beta)^{2} \partial_{w}, \\
& J_{3}(w)=(\alpha w+\beta)^{2} \partial_{w w}, \\
& J_{4}(w)=(\alpha w+\beta) \partial_{w w}-\alpha(1+n \gamma(\alpha w+\beta)) \partial_{w}, \\
& J_{5}(w)=\partial_{w w}-\frac{2 \alpha}{(\alpha w+\beta)} \partial_{w}-2 n \alpha \gamma \partial_{w}+\frac{2 n \alpha^{2} \gamma}{(\alpha w+\beta)}, \\
& J_{6}(w)=(\alpha w+\beta)^{2} \partial_{w}-n \alpha(\alpha w+\beta)+\frac{(\alpha w+\beta)}{\gamma} \partial_{w}, \\
& J_{7}(w)=1 .
\end{aligned}
$$

Proof. According to Proposition 6] a different basis for $\mathcal{M}_{n}(w)$ is given by

$$
\left\{y+\frac{1}{n \gamma}, y^{2}, \ldots, y^{n} \mid y=\alpha w+\beta\right\},
$$


and a straightforward computation will verify that the set of operators

$$
\begin{aligned}
& y^{4} \partial_{y y}+2(1-n) y^{3} \partial_{y}+n(n-1) y^{2}, \\
& y^{3} \partial_{y y}+(1-n) y^{2} \partial_{y}, \\
& y^{2} \partial_{y y}, \\
& y \partial_{y y}-(1+n \gamma y) \partial_{y}, \\
& \partial_{y y}-2 y^{-1} \partial_{y}-2 n \gamma \partial_{y}+\frac{2 n \gamma}{y}, \\
& y^{2} \partial_{y}-n y+\frac{y}{\gamma} \partial_{y},
\end{aligned}
$$

preserve the vector space spanned by (154). Making the appropriate substitutions yields directly (53). QED

\subsection{An example: the modified QES Morse potential}

Consider the following linear combination of the operators (53):

$$
T(z)=8 J_{3}(z)-8 \sqrt{2} J_{4}(z)+4 J_{5}(z)-16 J_{6}(z) .
$$

If we choose $\alpha=\beta=\delta=1 / \sqrt{2}$ and $\gamma=-1 / \sqrt{2}$ the above operator can be written in the form (15) with

$$
\begin{aligned}
& P(z)=4 z^{2} \\
& Q(z)=-4\left(2 z^{2}+(n+1) z-4+\frac{2}{1+z}\right) \\
& R(z)=8 n(1+z)-\frac{4 n}{1+z} .
\end{aligned}
$$

The change of variables determined by (17) is $z=\mathrm{e}^{2 x}$ and the potential is defined and free of singularities for all values of $x$. In particular, the potential form determined by (19) with (56)-(58) is

$$
U_{n}(x)=\frac{1}{8}\left(\cosh 4 x-n \cosh 2 x+\frac{1}{\left(1+\mathrm{e}^{2 x}\right)^{2}}-\frac{1}{1+\mathrm{e}^{2 x}}\right)
$$

where an additive constant has been dropped. If the last two terms were absent, this potential would be a QES deformation of the Morse potential, which belongs to the classification of Lie-algebraic potentials performed in 


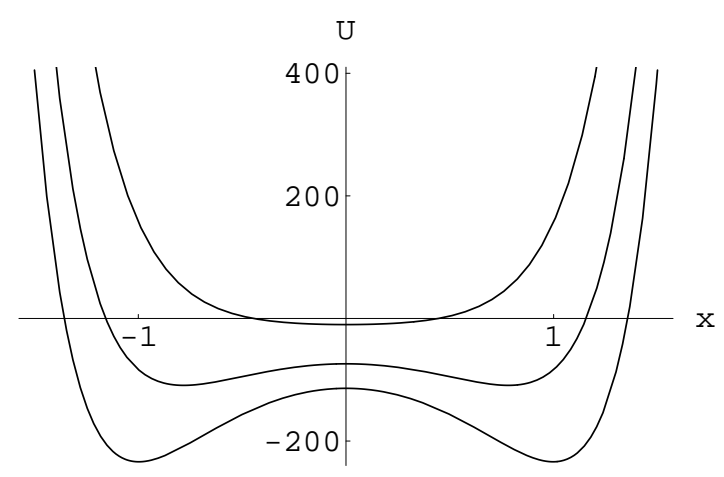

Figure 2: The deformed Morse potential $U_{n}(x)$ in (59) for $n=2,10$ and 15 .

[11]. A plot of this potential form for different values of $n$ can be found in Figure 2. When $n$ is a non-zero integer, $n$ eigenfunctions of the potential (59) can be calculated algebraically. They have the following form:

$$
\psi_{k-1}(x)=\mu(x) p_{k}\left(\mathrm{e}^{2 x}\right), \quad k=1, \ldots, n
$$

where $\mu(x)$ is determined by (18) with (156)-(58) to be

$$
\mu(x)=\frac{\mathrm{e}^{-\left(\mathrm{e}^{-2 x}+\mathrm{e}^{2 x}+(n-1) x\right)}}{1+\mathrm{e}^{2 x}}
$$

and $p_{k}(z)$ is one of the $n$ polynomial eigenfunctions that the operator (55) has in the space

$$
\mathcal{M}_{n}(z)=\operatorname{span}\left\{z+\frac{n-2}{n},(z+1)^{2}, \ldots,(z+1)^{n}\right\} .
$$

To be more specific, let us calculate a few eigenfunctions explicitly. If we let $n=3$, the action of $T(z)$ with respect to the basis (62) of $\mathcal{M}_{3}(z)$ is given by:

$$
\left.T(z)\right|_{\mathcal{M}_{3}(z)}=\left(\begin{array}{ccc}
-12 & 6 \sqrt{2} & 0 \\
16 \sqrt{2} & 16 & 0 \\
0 & 8 \sqrt{2} & 36
\end{array}\right)
$$

and the three algebraic eigenfunctions have been plotted in Figure 3. It is immediately apparent that the three algebraic functions have 0, 1 and 3 nodes, and therefore one eigenfunction of the potential (the one with two nodes) 


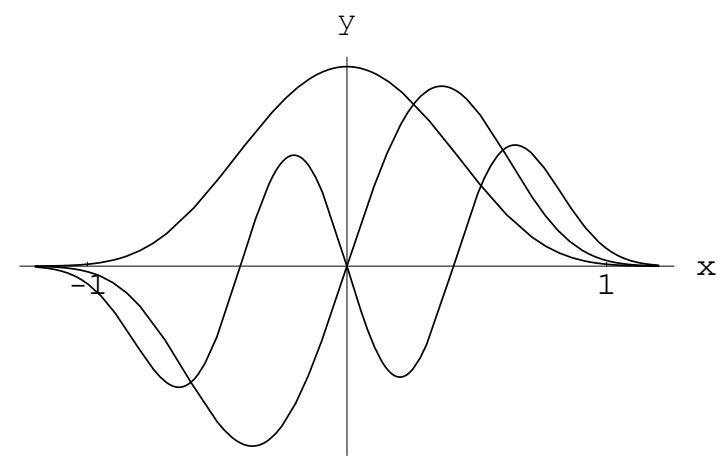

Figure 3: The three algebraic eigenfunctions $\psi_{0}, \psi_{1}$ and $\psi_{2}$ of $U_{3}(x)$.

is missing from the algebraic sector, i.e. it cannot have the factorized form (60). The situation is repeated for higher values of $n$ and our calculations suggest that in the general case, the eigenfunction with $n-1$ nodes does not belong to the algebraic sector. It remains an open question to find out what form this "missing" eigenfunction might have. This is the first example of a quasi-exactly solvable potential in which the algebraic eigenfunctions are not the lowest lying in the energy spectrum.

\section{Summary and conclusions}

We have shown how a more direct approach can be used to construct the most general differential operator of a given order $r$ that leaves invariant a polynomial subspace generated by monomials. This direct approach can be used to address other problems that would be very difficult to treat in the Liealgebraic approach, such as finding the most general differential operator that will map a polynomial module $\mathcal{P}_{n}$ into a proper subset $\mathcal{P}_{n-k} \subset \mathcal{P}_{n}$. Problems of this kind will be treated in our forthcoming publication [9], where this approach is also extended to deal with multivariate polynomials and nonlinear differential operators. The next step in difficulty involves extending the analysis to non-monomial modules (i.e. those which do not have a basis of monomials), a problem that remains still open. The applications of the direct approach to quasi-exact solvability in Quantum Mechanics point in two directions. First, they provide a very natural explanation of why some hamiltonians posses multiple algebraic sectors. As an example, we discuss 
a generalization of the Lamé potential (38), which could have interesting applications in solid state physics. Second, we emphasize that some quasiexactly solvable potentials exist which are not Lie-algebraic, as illustrated by the modified QES Morse potential (59). In this example there is always one bound state missing from the algebraic sector, which constitutes a new phenomenon in the theory of quasi-exactly solvable problems.

\section{acknowledgements}

The research of DGU is supported in part by a CRM-ISM Postdoctoral Fellowship and the Spanish Ministry of Education under grant EX2002-0176. The research of NK and RM is supported by the National Science and Engineering Research Council of Canada. DGU would like to thank the Department of Mathematics and Statistics of Dalhousie University for their warm hospitality.

\section{References}

[1] Alhassid, Y., Gürsey, F. and Iachello, F.: Potential scattering, transfer matrix and group theory, Phys. Rev. Lett. 50 873-876 (1983).

[2] Arscott, F. M.: Periodic Differential Equations, Oxford: Pergamon. 1964.

[3] Brihaye, Y. and Godard, G.: Quasi-exactly solvable extensions of the Lamé equation, J. Math. Phys. 34 5283-5291 (1993)

[4] Brihaye, Y. and Hartmann B.: Multiple algebraisations elliptic CalogeroSutherland model, J. Math. Phys. 441576 (2003)

[5] Finkel, F., González-Lopez, A. and Rodrígez, M. A.: A new algebraization of the Lamé equation, J. Phys. A 33 1519-1542 (2000)

[6] Finkel, F., González-Lopez, A., Maroto, A. L., and Rodrígez, M. A.: The Lamé equation in parametric resonance after inflation, Phys. Rev. D62103515 (2000)

[7] Finkel, F. and Kamran, N.: The Lie algebraic structure of differential operators admitting invariant spaces of polynomials, Adv. Appl. Math. 20, 300-322 (1998) 
[8] Gómez-Ullate, D., Gónzalez-López, A. and Rodríguez, M.A.: Exact solutions of an elliptic Calogero-Sutherland model, Phys. Lett. B511 112118 (2001)

[9] Gómez-Ullate, D., Kamran, N. and Milson, R.: Structure theorems for non-linear differential operators with invariant multivariate polynomial subspaces, in preparation.

[10] Gómez-Ullate, D., Kamran, N. and Milson, R.: The Darboux transformation and algebraic deformations of shape-invariant potentials, J. Phys. A 371789 - 1804 (2004)

[11] González-López, A., Kamran, N. and Olver, P.J.: Normalizability of one-dimensional quasi-exactly solvable Schrödinger operators, Commun. Math. Phys. 153 117-146 (1993)

[12] González-López, A., Kamran, N. and Olver, P.J.: Quasi-exact solvability, Contemp. Math. 160 113-140 (1994)

[13] González-López, A., Kamran, N. and Olver, P.J.: New quasi-exactly solvable Hamiltonians in two dimensions, Commun. Math. Phys. 159 503-537 (1994)

[14] Greene, P., Kofman, L., Linde, A. and Starobinsky, A.: Structure of resonance in preheating after inflation, Phys. Rev. D56 6175-6192 (1997)

[15] Kamran, N., Milson, R. and Olver, P.J.: Invariant modules and the reduction of nonlinear partial differential equations to dynamical systems, Adv. in Math. 156 286-319 (2000)

[16] Kamran, N. and Olver, P.J.: Lie algebras of differential operators and Lie-algebraic potentials, J. Math. Anal. Appl. 145 342-356 (1990)

[17] Olver, P. J.: Equivalence, Invariants, and Symmetry, Cambridge: Cambridge University Press. 1995.

[18] Post, G. and Turbiner, A. V.: Classification of linear differential operators with an invariant subspace of monomials, Russian J. Math. Phys. 3 113-122 (1995)

[19] Turbiner, A.V.: Quasi-exactly solvable problems and $\mathfrak{s l}(2)$ algebra, Commun. Math. Phys. 118, 467-474 (1988) 
[20] Turbiner, A.V.: Hidden algebra of the $N$-body Calogero problem, Phys. Lett. B320 281-286 (1994) 